\title{
Study of near-surface models in large-eddy simulations of a neutrally stratified atmospheric boundary layer
}

\author{
By I. Senocak, A.S. Ackerman $\dagger$, M.P. Kirkpatrick $\ddagger$, D.E. Stevens $\uparrow$ \\ AND N.N. Mansour
}

\section{Motivation and objectives}

Large-eddy simulation (LES) is a widely used technique in atmospheric modeling research, partly because of the difficulties involved in observational studies and field experiments to obtain information about the turbulent structure of the atmosphere (Stevens \& Lenschow 2001). In LES, large, unsteady, three dimensional structures are resolved and small structures that are not resolved on the computational grid are modeled. A filtering operation is applied to distinguish between resolved and unresolved scales. Unresolved motions are believed to be universal, and simple models should be sufficient to parameterize them, provided that a major fraction of the energetic large scales are resolved by the spatial and temporal resolution (Sagaut 2002). However, this requirement becomes stringent to fulfill in the close proximity of the surface/wall. Traditionally, the no-slip boundary has been referred to as "wall" in engineering context, and as "surface" in atmospheric science context. We will use the latter terminology throughout the paper.

As the surface is approached anisotropy in turbulence structure increases and the length scale of the flow structures diminish rapidly, requiring too fine a spatial and a temporal resolution to numerically resolve a large fraction of the energetic scales. Hence, fully resolved LES of atmospheric or any other wall-bounded high Reynolds number flow would be extremely expensive in terms of computational resources. In fact, it is this obstacle that motivates surface/wall modeling research in LES. Furthermore, the roughness of the surface underlying the atmospheric boundary layer has always been a complicated issue, and needs to be considered in modeling too.

In surface modeling, no-slip boundary conditions are not applied directly because the implied stress would be overestimated on a coarse grid. The simplest surface model that has long been adopted in atmospheric boundary layer simulations assumes that the logarithmic law holds within the surface layer, and stresses are imposed as boundary conditions at the surface(Schumann 1975; Moeng 1986). Cabot, Jimenez \& Baggett (1999) showed that such models have problems for high Reynolds number LES simulations with coarse numerical resolution. Cabot (1997) indicated that providing accurate mean surface stresses is not sufficient to overcome the overall poor predictions. He suggested that, given a coarse numerical resolution, typical subgrid-scale (SGS) models used in LES do not perform well to predict Reynolds stresses in the near-surface region accurately.

Boundary layer approximations have been proposed to extend the application of surface models for separating flows with adverse pressure gradient, (Balaras et al. 1996;

\footnotetext{
$\dagger$ NASA Ames Research Center

$\ddagger$ University of Tasmania

ণ Lawrence Livermore National Laboratory
} 
Cabot \& Moin 2000). In this approach, a simplified set of turbulent boundary layer equations, adopting a Reynolds-averaged Navier-Stokes (RANS) type eddy viscosity, are solved on an embedded mesh near the wall. The surface stress is then calculated from the computed velocity profile. Within this framework, Wang \& Moin (1992) utilized a dynamically adjusted mixing length eddy viscosity, and showed that their model performs significantly better than the simpler surface modeling approaches. Surface modeling for LES continues to be an active area of research for high Reynolds number flow, coarse resolution simulations. Latest research in this area focuses on incorporating suboptimal control theory to improve the predictive capability of the surface models (Templeton, Wang \& Moin 2002).

In atmospheric boundary layer simulations, the practice has been to employ LES models away from the surface and make a transition towards ensemble-averaged (RANS) models as the surface is approached. This idea has been adopted through different formulations. For instance, Sullivan et al. (1994) proposed an eddy viscosity model in which the so-called isotropy factor controls the transition from LES to a RANS type simulation, and accounts for the anisotropy effects at the same time. Mason \& Thomson (1992) introduced a modified length scale that is a matching between the LES filter size and the distance from the surface. Brown, Hobson \& Wood (2001) suggested using a canopy model with the aim of overcoming the difficulties in surface modeling. Traditionally, canopy models have been adopted to study the effect of flow within the canopy layer. In this approach, instead of modifying the eddy viscosity or the length scale, additional turbulent stresses are added to the turbulent stresses that are already modeled by the LES SGS model. Chow \& Street (2002) and Kirkpatrick et al. (2003) adopted the canopy model of Brown et al. (2001) for atmospheric boundary layer simulations, and reported improved predictions. We will refer to these models, in which additional stresses are added onto sugrid-scale models to improve the predictions in the vicinity of the surface, as "near-surface" models. We reserve the term "surface model" to include both the models for surface boundary condition and the near-surface models.

In what follows, we present two near-surface models that have found use in atmpspheric modeling. We also suggest a simpler eddy viscosity model that adopts Prandtl's mixing length model (Prandtl 1925) in the vicinity of the surface and blends in with the dynamic Smagorinsky model (Germano et al. 1991) away from the surface. We evaluate the performance of these surface model by simulating a neutrally stratified atmospheric boundary layer.

\section{Governing equations}

The governing equations for LES of a neutrally stratified atmospheric boundary layer are the filtered Navier-Stokes equations

$$
\frac{\partial \bar{u}_{i}}{\partial x_{i}}=0, \quad \frac{\partial \bar{u}_{i}}{\partial t}+\frac{\partial\left(\bar{u}_{i} \bar{u}_{j}\right)}{\partial x_{j}}=\frac{\partial \bar{p}}{\partial x_{i}}+\epsilon_{i j k} f_{j} \bar{u}_{k}-\frac{\partial \tau_{i j}}{\partial x_{j}},
$$

where turbulent stresses are defined as $\tau_{i j}=\overline{u_{i} u_{j}}-\bar{u}_{i} \bar{u}_{j}, f_{j}$ is the Coriolis parameter, $\epsilon_{i j k}$ is the permutation tensor, $\bar{u}$ and $\bar{p}$ are the filtered velocity and filtered dynamic pressure, respectively.

The lateral boundary conditions are periodic and a stress-free condition is imposed on the upper boundary. At the lower boundary, the vertical component of the velocity is set to zero and horizontal components of the turbulent stresses are defined based on the 
mean logarithmic wind profile assumption as follows (Moeng 1984)

$$
\tau_{i 3}=-\left(\frac{\kappa}{\ln \frac{z_{1}}{z_{o}}}\right)^{2}|u| \bar{u}_{i},
$$

where $z_{1}, u_{i}$ and $z_{0}$ are the vertical spacing, the velocity of the first grid point away from the surface, and the roughness height, respectively. This type of boundary condition for the surface is a common practice in atmospheric modeling.

\section{Subgrid-scale turbulence modeling}

Turbulent stresses that appear in equation 2.1 are modeled based on the Boussinesq eddy viscosity assumption. The Smagorinsky eddy viscosity model (Smagorinsky 1963) is a popular approach to represent turbulent stresses

$$
\tau_{i j}=-2 C l^{2}|\bar{S}| \bar{S}_{i j}
$$

where $|\bar{S}|$ is the magnitude of the filtered strain rate tensor, it is defined as

$$
|\bar{S}|=\sqrt{2 \bar{S}_{i j} \bar{S}_{i j}}, \quad \bar{S}_{i j}=\frac{1}{2}\left(\frac{\partial \bar{u}_{i}}{\partial x_{j}}+\frac{\partial \bar{u}_{j}}{\partial x_{i}}\right) .
$$

In LES, the filter width $\Delta=(d x \cdot d y \cdot d z)^{1 / 3}$ is chosen as the length scale $l$ in the eddy viscosity definition (equation 3.1). In the original Smagorinsky model, the dimensionless parameter $C$ is an empirical constant, whereas in the dynamic Smagorinsky model (Germano et al. 1991), this parameter is computed dynamically during the solution, making it a function of space and time.

In the vicinity of the surface, the numerical resolution is generally limited and nearsurface models are adopted to model the turbulent stresses properly. We consider three near-surface models, and provide a brief explanation of them in the following.

\subsection{Mason and Thomson Model}

This is an arbitrary and simple modification of the length scale in the eddy viscosity definition given by equation 3.1. Mason \& Thomson (1992) have suggested using the following form as the length scale.

$$
\frac{1}{l^{2}}=\frac{1}{\Delta^{2}}+\frac{1}{(\kappa z)^{2}}
$$

where $\kappa$ is the von Karman constant with a value of 0.41 , and $z$ is the distance from the surface.

\subsection{Canopy Stress Model}

Canopy models are usually adopted to model the structure of the flow within the canopy. Brown et al. (2001) have suggested using them as near-surface models in the case of coarse numerical resolution. In particular, we follow the implementation of Kirkpatrick et al. (2003). Canopy stresses near the surface are formulated as

$$
\tau_{i 3}=-\int_{0}^{h} C_{s} \cos \left(\frac{\pi z}{2 h}\right)|u| u_{i} d z
$$

where $C_{s}$ is a normalizing parameter. Specifically, $C_{s}$ is the ratio of the surface stress at the bottom to the canopy stress at the first grid point. The canopy height $h$ is taken to 
be twice the streamwise grid spacing $(2 d x)$. Canopy stresses are added onto the turbulent stresses that are modeled with the dynamic Smagorinsky model.

\subsection{Hybrid RANS/LES model}

We adopt a hybrid RANS/LES approach as the third model. The motivation behind this model is that the surface stress boundary conditions represent ensemble-averaged quantities, because they are derived from the mean logarithmic wind profile. Hence, it is more meaningful to adopt a RANS type eddy viscosity formulation near the surface.

One can analytically show that the logarithmic wind profile can be derived from the following eddy viscosity definition (Panofsky \& Dutton 1984)

$$
\nu_{t}=\kappa u_{*} z
$$

where $u_{*}$ is the friction velocity. The above form is also identical to the following definition

$$
\nu_{t}=(\kappa z)^{2} \frac{\partial U}{\partial z}=(\kappa z)^{2}|S| .
$$

Here, $U$ is magnitude of the velocity vector. We apply the above definition in the vicinity of the surface, which guarantees a logarithmic wind profile, and switch to the dynamic Smagorinsky model away from the surface with the help of a blending function. This hybrid RANS/LES model can be written as

$$
\nu_{t}=\left[(1-\exp (-z / h))^{2} \cdot\left(C \Delta^{2}\right)+\exp (-z / h)^{2}(\kappa z)^{2}\right]|S|
$$

Following the suggestion of van Driest (1956) an exponential form is selected as the blending function. Here $h$ is the altitude, where the logarithmic wind profile is not expected further. As a rule of thump, a logarithmic velocity profile is expected within the surface layer, which is the bottom 10 percent of the atmospheric boundary layer height $(100 \mathrm{~m}$ $-200 \mathrm{~m}$ ) (Stull 1988). $h=150 \mathrm{~m}$ is used for the present computations.

\section{Numerical schemes}

We use the LES atmospheric research code (DHARMA) for the present simulations. The numerical method adopted in DHARMA is described in detail in Stevens \& Bretherton (1996) and Stevens et al. (2000). The governing equations are integrated using a forward-in-time projection method based on an explicit second-order Runge-Kutta scheme (Bell \& Marcus 1992). The spatial discretization is performed on a staggered grid. A third-order accurate upwind-biased monotonic scheme is used for the advection terms, whereas diffusion and pressure gradient terms are discretized using second-order accurate central differencing schemes. A direct solver (FFT) is utilized for solving the

pressure Poisson equation, and the code has been parallelized to run on various platforms using MPI.

\section{Results}

In this section, we present results from the simulation of neutrally stratified atmospheric boundary layer. The computational domain size is $3000 \mathrm{~m} \times 1500 \mathrm{~m} \times 1500$ $\mathrm{m}$ with $64 \times 32 \times 64$ grid points in $\mathrm{x}$, $\mathrm{y}$, and $\mathrm{z}$ directions, respectively. In the vertical direction, the grid points are clustered exponentially near the surface, where the first grid point for the horizontal component of the velocity is located at $4 \mathrm{~m}$ from the surface. The flow is driven by the Coriolis force that would balance a $10 \mathrm{~ms}^{-1}$ geostrophic 


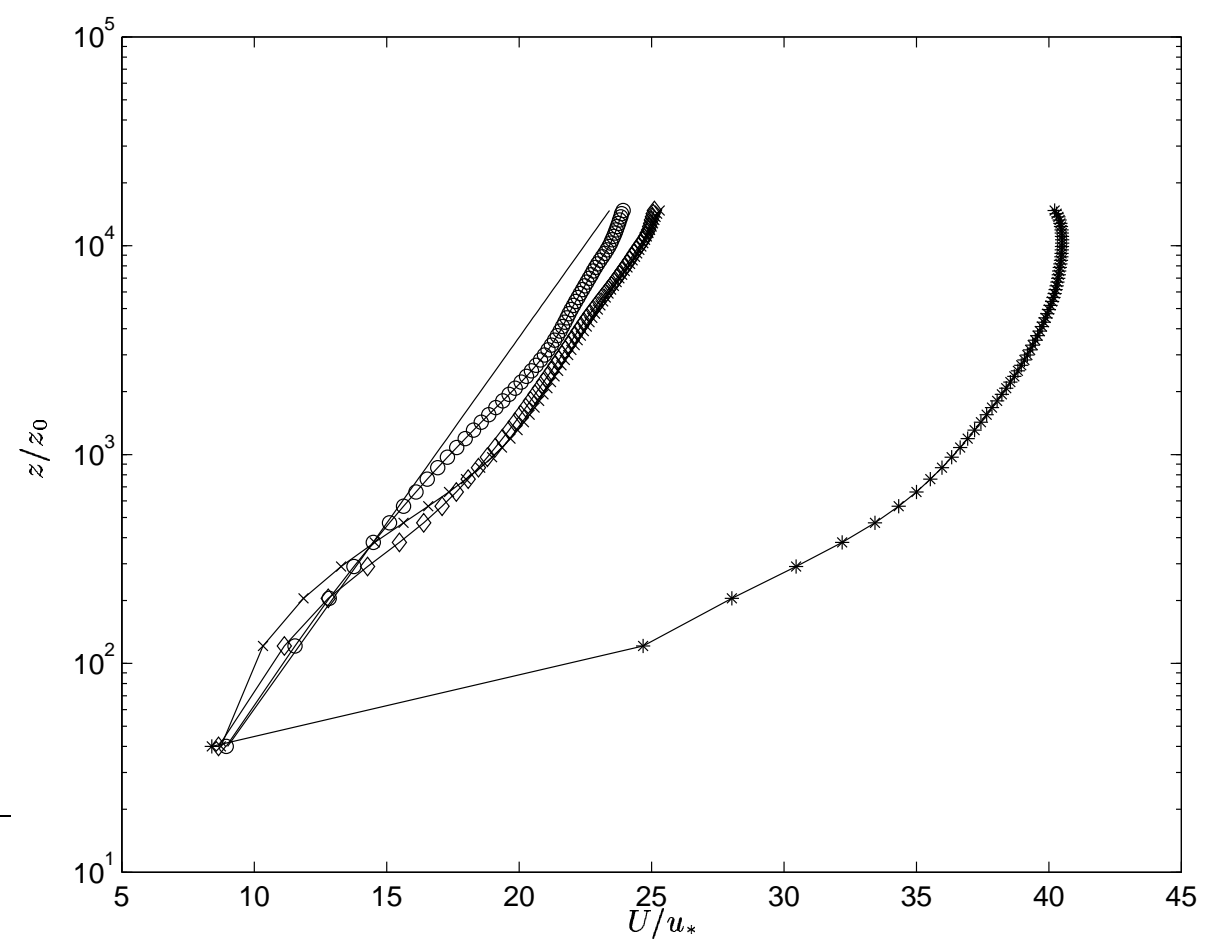

Figure 1. Comparison of mean wind profile. —— : log-law, - - - : no near-surface model, $\longrightarrow \circ-$ : hybrid RANS/LES model, $\longrightarrow$ : canopy stress model, $-\times-$ : Mason-Thomson model

wind in the $\mathrm{x}$ direction. Coriolis parameter is set equal to $10^{-4} \mathrm{~s}^{-1}$, and the roughness parameter $z_{0}$ has a value of $0.1 \mathrm{~m}$. A dimensionless time unit can be defined based on the Coriolis parameter $(1 / f)$. The simulations were run over a period of $10 f^{-1}$, and statistics are collected during the time period of last the $4 f^{-1}$. Ensemble averaged vertical profiles have been obtained by collecting data at every $6 \times 10^{-3}$ dimensionless units (60secs), and averaging them both in time and in horizontal space.

Andren et al. (1994) compared the performance of different LES computer codes to simulate a neutrally stratified atmospheric boundary layer. They concluded that results were more sensitive to the SGS model in the lower third of the boundary layer, and commonly used SGS models have failed to reproduce the logarithmic wind profile in the vicinity of the surface. Hence, satisfying the logarithmic wind profile near the surface has become one of the measures of good performance for an SGS model.

Figure 1 shows a comparison of the mean wind profiles for all the models considered in the present study. When no near-surface model is used, the dynamic Smagorinsky model produces an erroneous profile. This indicates that providing only the surface stress boundary condition is not sufficient to model the SGS motions near the surface in the case of high Reynolds number and coarse numerical resolution. This observation is in agreement with the findings of Cabot et al. (1999). It should be noted that the erroneous profile is not surprising because the essence of the dynamic Smagorinsky model is based on resolving the inertial subrange. This has not been satisfied with the current coarse 

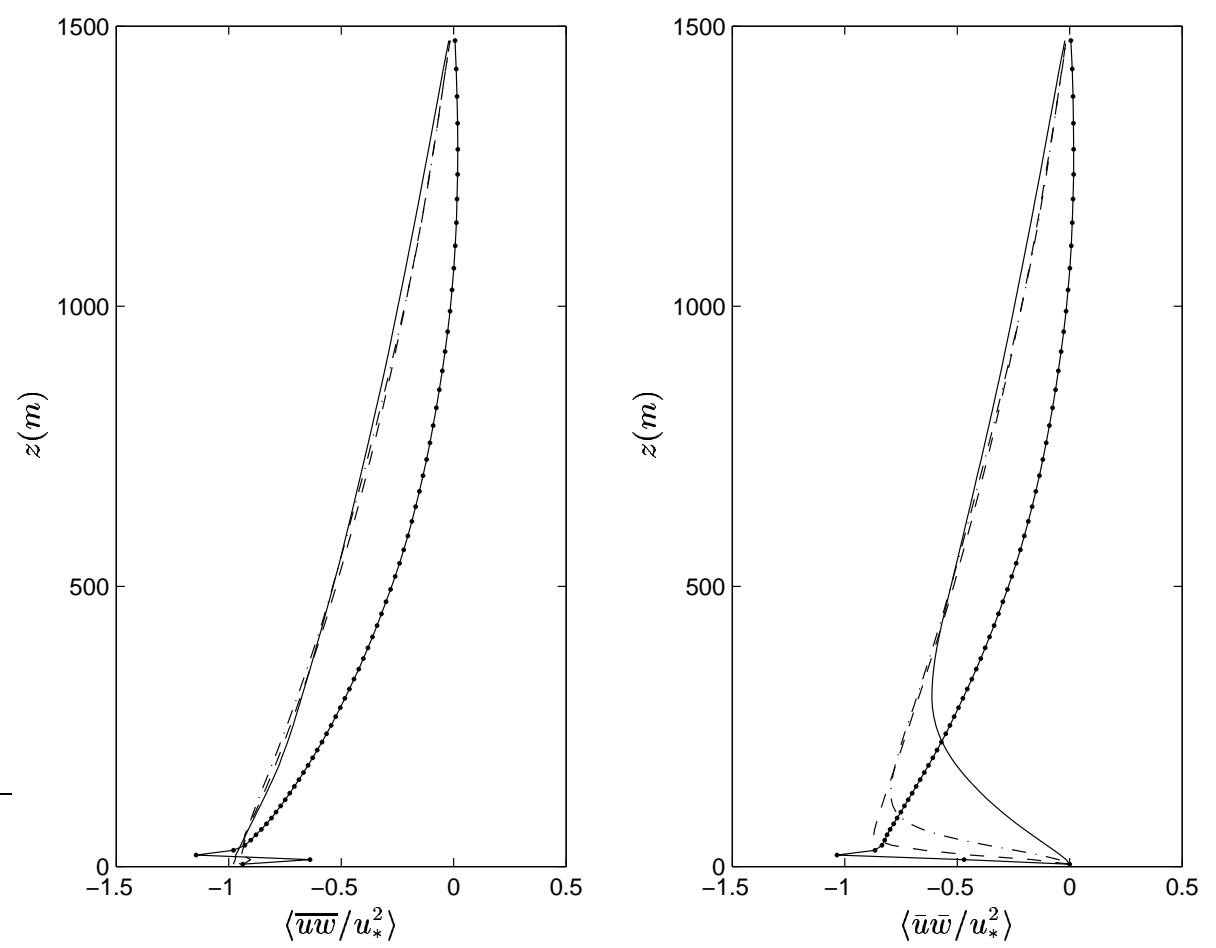

FIGURE 2. Comparison of streamwise component of the total (left) and resolved (right) stress. ... : no near-surface model, — : hybrid RANS/LES model, —- : canopy stress model, -. - : Mason-Thomson model

resolution. Hence, SGS models need augmentation to represent the unresolved scales near the surface.

The near-surface models considered in the present study, namely, Mason-Thomson model, the canopy stress model and the hybrid RANS/LES model are formulated to parameterize the unresolved scales near the surface. Both the canopy stress model and the hybrid RANS/LES model adopt the dynamic Smagorinsky model away from the surface, whereas the Mason-Thomson model adopts the original Smagorinsky model. As seen from figure 1, results obtained from these three near-surface models are much closer to the loglaw profile. However, considerable differences exist among them. The best agreement with the log-law near the surface is achieved with the hybrid RANS/LES model, whereas the Mason and Thomson model gives the worst agreement. The hybrid RANS/LES model has the closest agreement, because the length scale is taken to be the distance from the surface in the model, which is known to give the log-law analytically. The canopy stress model follows the log-law close to the surface, but a significant deviation is observed further from the surface. This indicates that, canopy stresses should be distributed over a larger distance from the surface. We reiterate that, based on field experiments, a logarithmic velocity profile is expected in the surface layer, which is approximately the bottom 10 percent of the atmospheric boundary layer height $(100 \mathrm{~m} \mathrm{-200} \mathrm{m).}$

The streamwise $\left(\left\langle\overline{u w} / u_{*}^{2}\right\rangle\right)$ component of the total and resolved turbulent stress profiles are compared in figure 2. By total stress, we mean the resolved turbulent stresses plus the modeled SGS turbulent stresses. As seen from this plot, simulations with surface models predict an almost linear variation of the total turbulent stress in agreement with surface 

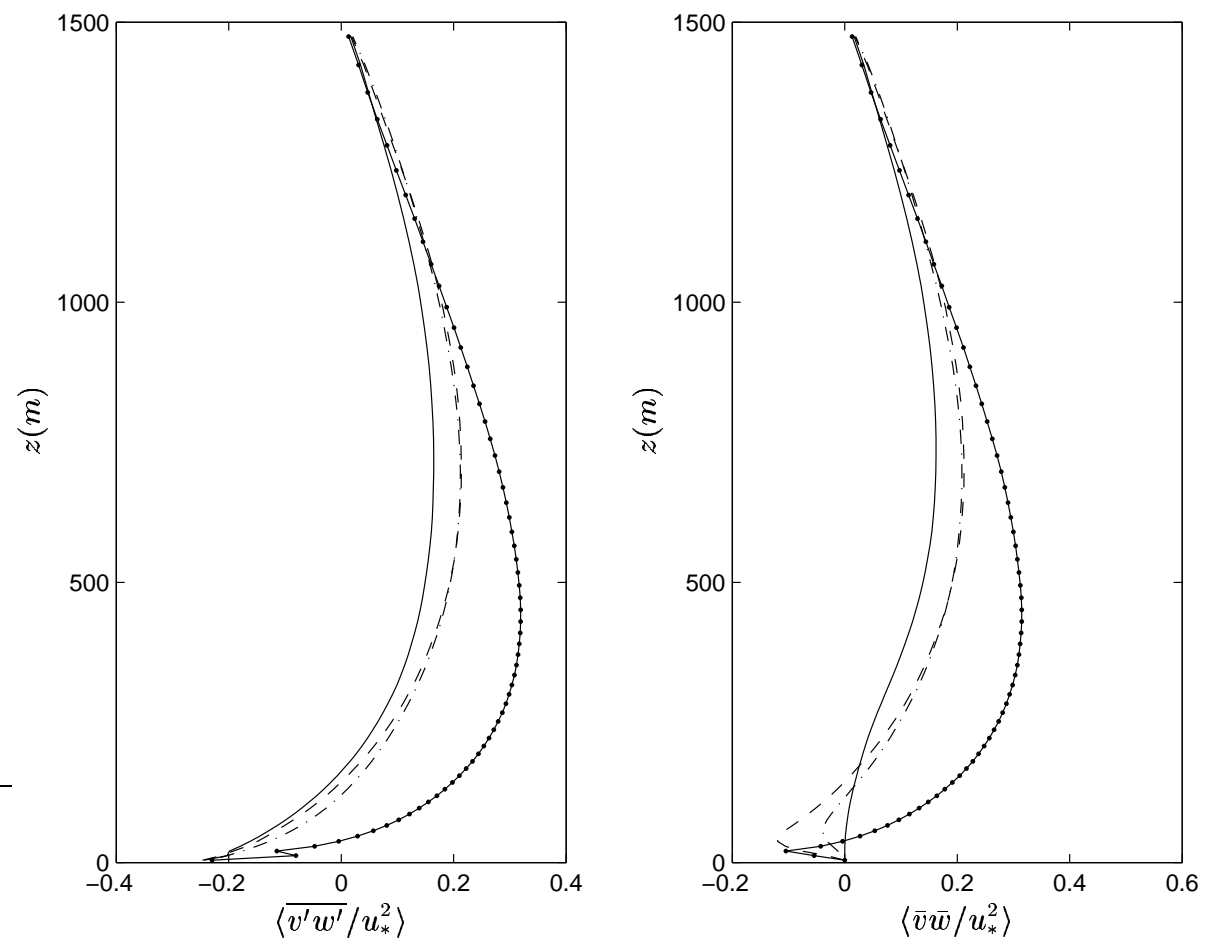

FigURE 3. Comparison of spanwise component of the total (left) and resolved (right) stress. ‥ : no near-surface model, — : hybrid RANS/LES model, ——— : canopy stress model, $-\cdot-$ : Mason-Thomson model

similarity theory (Garratt 1992). However, without a near-surface model, the dynamic Smagorinsky model does not predict a linear variation of the total turbulent stress and produces wiggles at the surface, indicating the need for near-surface modeling.

The resolved turbulent stresses are also shown in figure 2. Within the lower one fourth of the boundary layer, a large range of turbulent scales are modeled and not resolved with the hybrid RANS/LES model, whereas other surface models are effective only within the lower one tenth of the boundary layer. The extent of RANS modeling in the hybrid RANS/LES model is adjustable through the value of $h$ in equation 3.7. We have found that $h=150 \mathrm{~m}$ is reasonable in terms of satisfying the logarithmic wind profile in accordance with experimental observations.

The differences among the three surface models are more pronounced in the spanwise $\left(\left\langle\overline{v^{\prime} w^{\prime}} / v_{*}^{2}\right\rangle\right)$ component of the total stress as shown in 3 . This spanwise component of the total stress is generated due to the rotation of the Earth. The variation of the velocity vector with height is known as the Ekman spiral (Stull 1988).

As can be seen from figure 4, the spanwise component of the velocity is also affected because of differences in the distribution of the spanwise component of the total turbulent stress. With all three surface models, we see that the spanwise component of the velocity does not vanish at the top, whereas it vanishes in the case of the dynamic Smagorinsky model without a near-surface model. Apparently, nonvanishing spanwise velocity at the top is an outcome of employing the surface models. Since ensemble averaged quantities are obtained near the wall, fluctuations of the velocity field are damped due to the action of turbulent eddy viscosity or canopy stresses, effectively resulting in a laminar flow field. 


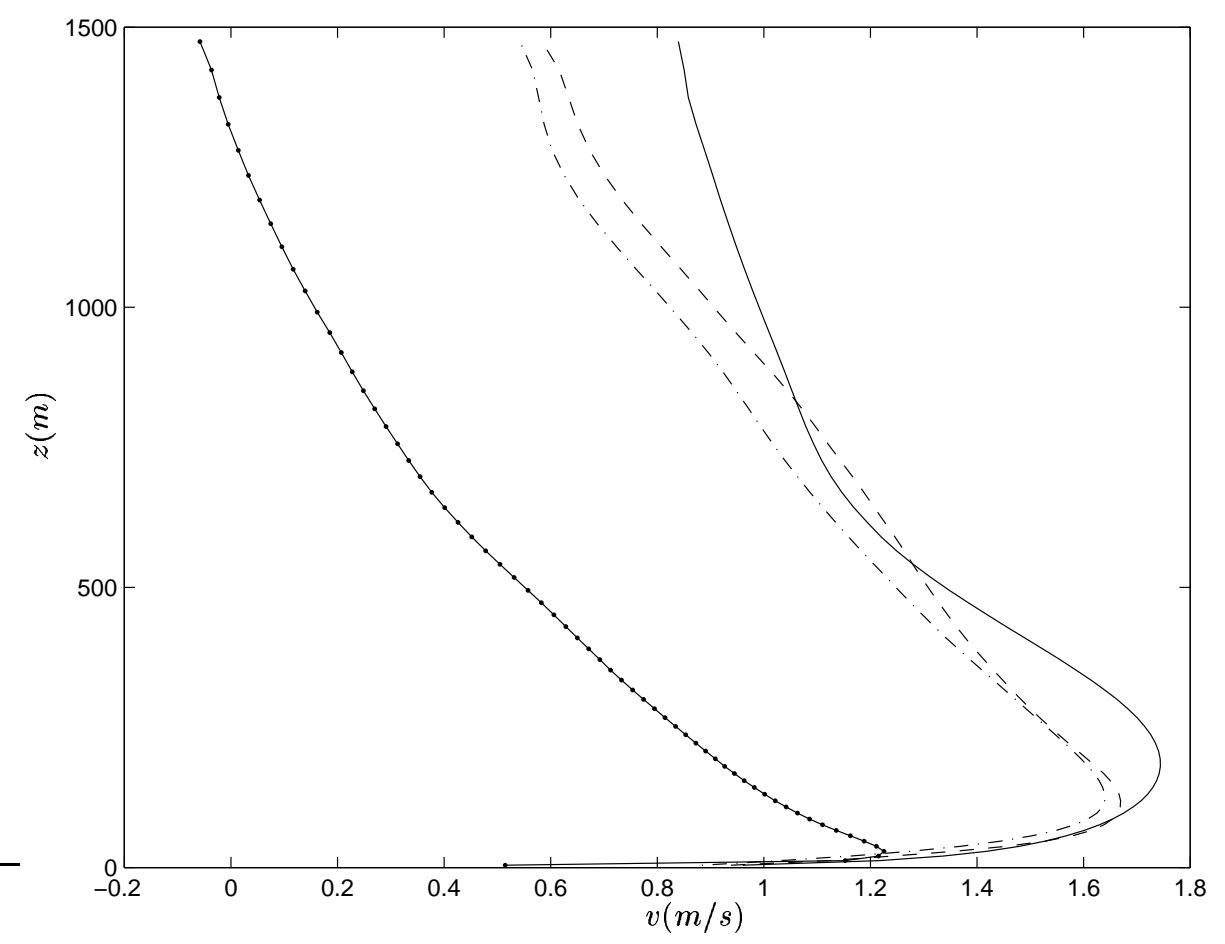

FIGURE 4. Comparison of spanwise component (v) of the velocity profiles. $\cdots$ : no near-surface model, $\longrightarrow$ : hybrid RANS/LES model, ——— : canopy stress model, $-\cdot$ - : Mason-Thomson model

As a result, this ensemble averaged region acts like an artificial boundary layer for the flow field away from the surface that is modeled in the LES sense. Baggett (1998) have reported similar issues in LES of channel flow.

We present turbulent eddy viscosity profiles in figure 5. Away from the surface, identical profiles are obtained with the hybrid RANS/LES model and the canopy stress model. Near the surface, the eddy viscosity levels are much lower with the canopy stress model. This is because the model modifies the stress terms but not the eddy viscosity. On the other hand, eddy viscosity level is much higher near the surface with the hybrid RANS/LES model. With such high levels of eddy viscosity, turbulent motions near the surface are dampened, resulting in an ensemble averaged velocity field. Among all models, the Mason-Thomson predicts higher levels of eddy viscosity away from the surface, because it adopts the original Smagorinsky model in that region.

\section{Summary and conclusions}

In this study, we have performed large-eddy simulations of a neutrally stratified atmospheric boundary layer. Particularly, we have assessed the performance of different near-surface models in predicting the mean flow structure. The surface of our atmosphere is always rough and typical numerical resolutions that are utilized in atmospheric boundary layer modeling are too coarse near the surface to perform well-resolved largeeddy simulations. This necessitates additional modeling in the vicinity of the surface to take into account the unresolved turbulent flow, covering a large range of scales. We have 


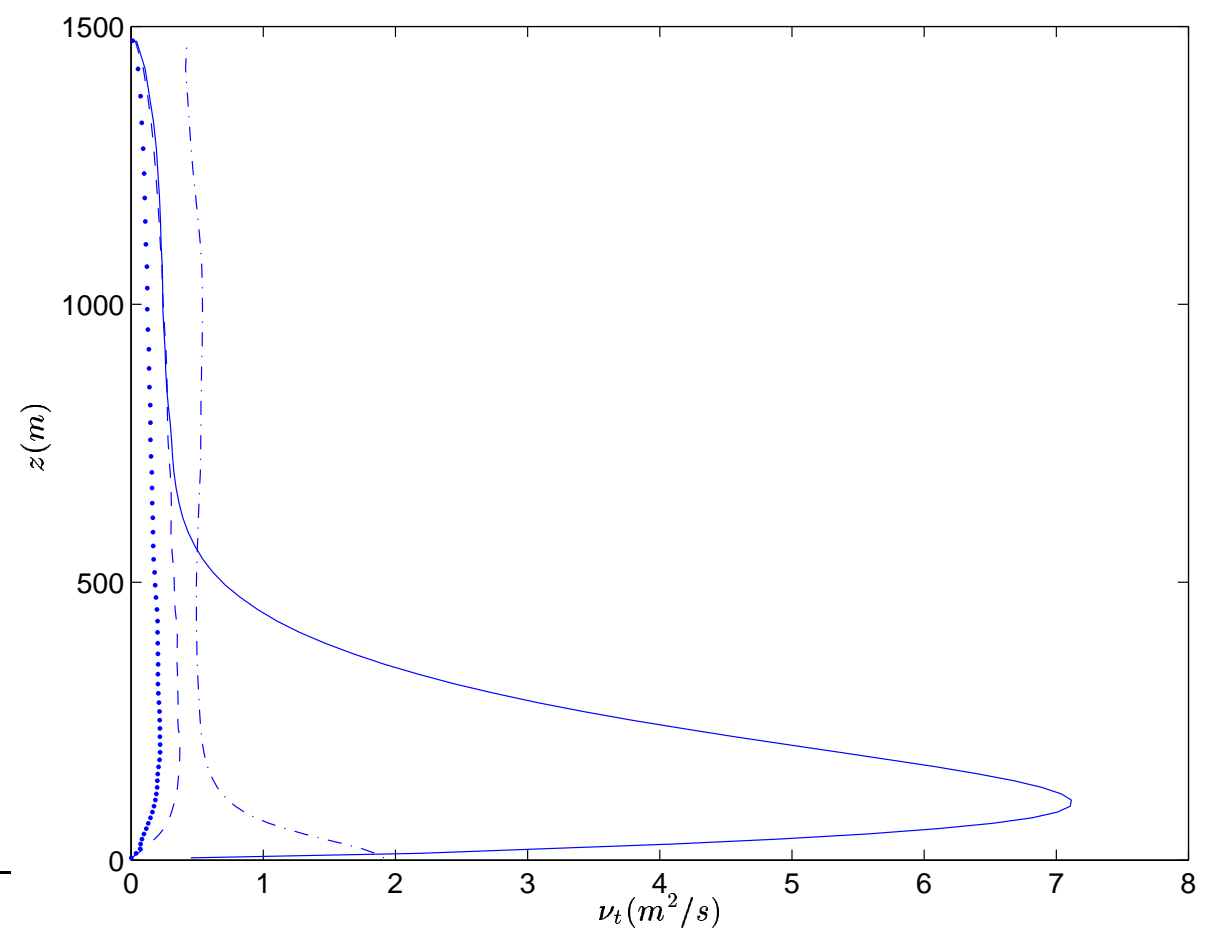

FiguRE 5. Comparison of turbulent eddy viscosity profiles. ‥ : no near-surface model, — : hybrid RANS/LES model, _- — : canopy stress model, $-\cdot-$ : Mason-Thomson model

considered the near-surface models of Mason \& Thomson (1992), Brown et al. (2001) and also suggested a hybrid RANS/LES model that blends Prandtl's mixing length model with the dynamic Smagorinsky model. We have also considered the dynamic Smagorinsky model with no near-surface model to highlight the need for surface modeling in the case of coarse numerical resolution.

Off all the near-surface models considered, the hybrid RANS/LES model shows better agreement with mean logarithmic wind profile. In the canopy stress model, it appears that surface stresses should be distributed to a larger distance from the surface to improve the predictions. The downside of adopting any of the near-surface models is that the spanwise component of the velocity vector does not vanish at the top of the computational domain. For the current numerical resolution with the hybrid RANS/LES model, the contribution from RANS modeling is effective on about the lower one fourth of the domain. A finer numerical resolution might help confine the RANS contribution very close to the surface and get better predictions at the same time.

Although comparable predictions can be obtained by either adopting the hybrid RANS/LES or the canopy stress model, the hybrid RANS/LES model has the advantage of easy implementation. In a parallel effort (Senocak et al. 2004), we are implementing the immersed boundary method in DHARMA. We find that it is much more convenient to implement the hybrid RANS/LES model to simulate flow over topography with the immersed boundary method because only the eddy viscosity term needs modification. However, it might not be straightforward to adopt the canopy stress model in Cartesian grid techniques for complex topography simulations. 
Andren, A., Brown, A. R., Graf, J., Mason, P. J., Moeng, C.-H., Nieuwstadt, F. T. M. \& Schuman, U. 1994 Large-eddy simulation of a neutrally stratified boundary layer: A comparison of four computer codes. Q. J. R. Meteorol. Soc. 120, 1457-1484.

BAGGETT, J. S. 1998 On the feasibility of merging LES with RANS for the near-wall region of attached turbulent flows. Annual Research Briefs, Center for Turbulence Research, NASA-Ames/Stanford Univ.

Balaras, E., Benocci, C. \& Piomelli, U. 1996 Two-layer approximate boundary conditions for large-eddy simulation. AIAA J. 34, 1111-1119.

BeLL, J. B. \& MARCus, D. L. 1992 A second-order projection method for variabledensity flows. J. Comp. Phys. 101, 334-348.

Brown, A. R., Hobson, J. M. \& Wood, N. 2001 Large-eddy simulation of neutral turbulent flow over rough sinusoidal ridges. Boundary-Layer Meteorol. 98, 411-441.

САвот, W. 1997 Wall models in large-eddy simulation of seperated flow. Annual Research Briefs, Center for Turbulence Research, NASA-Ames/Stanford Univ.

Савот, W., Jimenez, J. \& Baggett, J. S. 1999 On wakes and near-wall behavior in coarse large-eddy simulation of channel flow with wall models and second-order finite difference methods. Annual Research Briefs, Center for Turbulence Research, NASA-Ames/Stanford Univ.

Савот, W. \& Moin, P. 2000 Approximate wall boundary conditions in the large-eddy simulation of high Reynolds number flow. Flow, Turbul. Combust. 63, 269-291.

Chow, F. K. \& Street, R. 2002 Modeling unresolved motions in LES of field-scale flows. In 15th Symposium on Boundary Layers and Turbulence.

van Driest, E. R. 1956 On turbulent flow near a wall. J. Aero. Sci. 23, 1007-1011.

Garratt, J. R. 1992 The Atmospheric Boundary Layer. Cambridge University Press.

Germano, M., Piomelli, U., Moin, P. \& Cabot, W. H. 1991 A dynamic subgridscale eddy viscosity model. Phys. Fluids 3, 1760-1765.

Kirkpatrick, M. P., Mansour, N. N., Ackerman, A. S. \& Stevens, D. E. 2003 Dynamic turbulence modeling in large-eddy simulations of the cloud-topped atmospheric boundary layer. Annual Research Briefs, Center for Turbulence Research, NASA-Ames/Stanford Univ.

Mason, P. \& Thomson, D. 1992 Stochastic backscatter in large-eddy simulations of boundary layers. J. Fluid Mech. 242, 51-78.

Moeng, C. H. 1984 A large-eddy simulation model for the study of planetary boundarylayer turbulence. J. Atmos. Sci 41, 2052-2062.

Moeng, C. H. 1986 Large-eddy simulation of a stratus-topped boundary layer. Part-1: Structure and budgets. J. Atmos. Sci 43, 2886-2900.

Panofsky, H. A. \& Dutton, J. A. 1984 Atmospheric Turbulence. John Wiley.

Prandth, L. 1925 Bericht uber die entstehung der turbulenz. Z. Angew. Math. Mech. 5, 136-139.

Sagaut, P. 2002 Large Eddy Simulation for Incompressible Flows. Springer-Verlag.

Schumann, U. 1975 Subgrid-scale model for finite difference simulations of turbulent flows in plane channels and annuli. J. Comp. Phys. 18, 376-404.

Senocak, I., Ackerman, A. S., Stevens, D. E. \& Mansour, N. N. 2004 Topogra- 
phy modeling in atmospheric flows using the immersed boundary method. Annual Research Briefs, Center for Turbulence Research, NASA-Ames/Stanford Univ.

SMAgorinsky, J. 1963 General circulation experiments with the primitive equations. I. The basic experiment. Mon. Weather Rev. 91, 99-164.

Stevens, B. \& Lenschow, D. H. 2001 Observations, experiments, and large eddy simulation. Bull. Amer. Meteor. Soc. 82, 283-294.

Stevens, D. E., Bell, J. B., Almgren, A. S., Beckner, V. E. \& Rendleman, C. A. 2000 Small-scale processes and entrainment in a stratocumulus marine boundary layer. J. Atmos. Sci. 57, 567-581.

Stevens, D. E. \& Bretherton, C. S. 1996 A forward-in-time advection scheme and adaptive multilevel flow solver for nearly incompressible atmospheric flow. J. Comp. Phys. 129, 284-295.

StulL, R. B. 1988 An Introduction to Boundary Layer Meteorology. Kluwer Academic Publishers.

Sullivan, P. P., McWilliams, J. C. \& Moeng, C. H. 1994 A subgrid-scale model for large-eddy simulation of planetary boundary layer flows. Boundary-Layer Meteorol. 71, 247-276.

Templeton, J. A., Wang, M. \& Moin, P. 2002 Towards LES wall models using optimization techniques. Annual Research Briefs, Center for Turbulence Research, NASA-Ames/Stanford Univ.

WANG, M. \& Moin, P. 1992 Dynamic wall modeling for large-eddy simulation of complex turbulent flows. Phys. Fluids 14, 2043-2051. 